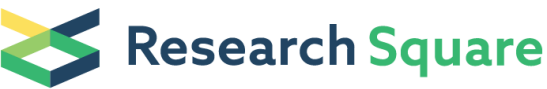 \\ Preprints are preliminary reports that have not undergone peer review. \\ They should not be considered conclusive, used to inform clinical practice, or referenced by the media as validated information.
}

\section{Thermal disorder driven magnetic phases in van der Waals magnet $\mathrm{Cr} 33$}

\section{Jaume Sanches}

University of Valencia

\section{Dina Wahab}

Queen's University Belfast

Hubertus Luetkens

Paul Scherrer Institute

\section{Grigol Taniashvili}

Tbilisi State University

\section{Efren Moratalla}

Instituto de Ciencia Molecular, Universitat de Valencia, Calle Catedratico JoseBeltran Martınez

\section{Zurab Guguchia}

Laboratory for Muon Spin Spectroscopy, Paul Scherrer Institute, CH-5232 Villigen PSI, Switzerland https://orcid.org/0000-0002-5498-328X

Elton Santos ( $\square$ esantos@ed.ac.uk)

The University of Edinburgh https://orcid.org/0000-0001-6065-5787

\section{Article}

Keywords: Thermal disorder driven magnetic phases, van der Walls (vdW) magnetic materials, Crl3

Posted Date: October 6th, 2020

DOI: https://doi.org/10.21203/rs.3.rs-81635/v1

License: (c) (1) This work is licensed under a Creative Commons Attribution 4.0 International License. Read Full License

Version of Record: A version of this preprint was published at Nature Communications on November 1st, 2021. See the published version at https://doi.org/10.1038/s41467-021-26342-4. 


\section{Thermal disorder driven magnetic phases in van der Waals magnet $\mathrm{Crl}_{3}$}

3 Jaume Meseguer-Sánchez ${ }^{1}$, Dina Abdul Wahab ${ }^{2}$, Hubertus Luetkens ${ }^{3}$, Grigol Taniashvili ${ }^{4}$, Efrén

4 Navarro-Moratalla ${ }^{1, \dagger}$, Zurab Guguchia ${ }^{3, \dagger}$, Elton J. G. Santos ${ }^{5, \dagger}$

${ }_{5}{ }^{1}$ Instituto de Ciencia Molecular, Universitat de Valéncia, Calle Catedrático José Beltrán Martínez

6 2, 46980, Paterna, Spain

$7{ }^{2}$ School of Mathematics and Physics, Queen's University Belfast, BT7 1NN, United Kingdom

${ }_{8}^{3}$ Laboratory for Muon Spin Spectroscopy, Paul Scherrer Institute, CH-5232 Villigen PSI, Switzer-

9 land

${ }_{10}{ }^{4}$ Department of Physics, Tbilisi State University, Chavchavadze 3, GE-0128 Tbilisi, Georgia

${ }_{11}^{5}$ Institute for Condensed Matter Physics and Complex Systems, School of Physics and Astronomy,

12 The University of Edinburgh, EH9 3FD, UK

${ }_{13}^{\dagger}$ Correspondences to: efren.navarro@me.com, zurab.guguchia@psi.ch, esantos@ed.ac.uk

Magnetic phase transitions often occur spontaneously at specific critical temperatures and are instrumental to understand the origin of long-range spin order in condensed matter systems. The presence of more than one critical temperature $\left(T_{c}\right)$ has been observed in several compounds $\mathrm{s}^{1 \cdot \sqrt[5]{5}}$ where the coexistence of competing magnetic orders highlights the importance of phase separation driven by different factors such as pressure, temperature and chemical composition. However, it is unknown whether recent discovered two-dimensional (2D) van der Walls (vdW) magnetic materials ${ }^{67}$ show such intriguing phenomena that can result in 
rich phase diagrams with novel magnetic features to be explored. Here we show the existence of three magnetic phase transitions at different $T_{c}$ 's in $2 \mathrm{D}$ vdW magnet $\mathrm{CrI}_{3}$ revealed by a complementary suite of muon spin relaxation-rotation $(\mu \mathrm{SR})$, superconducting quantum interference device (SQUID) magnetometry, and large-scale micromagnetic simulations including higher-order exchange interactions and dipolar fields ${ }^{8}$. We find that the traditionally identified Curie temperature of bulk $\mathrm{CrI}_{3}$ at $61 \mathrm{~K}^{\mathbf{9}}$ does not correspond to the longrange order in the full volume $\left(V_{M}\right)$ of the crystal but rather a partial transition with less than $\sim 25 \%$ of $V_{M}$ being magnetically spin-ordered. This transition is composed of highlydisordered domains with the easy-axis component of the magnetization $\left(S_{z}\right)$ not being fully spin-polarized but disordered by in-plane components $\left(S_{x}, S_{y}\right)$ over the entire layer. As the system cools down, two additional phase transitions at $50 \mathrm{~K}$ and $25 \mathrm{~K}$ drive the system to $80 \%$ and nearly $100 \%$ of the magnetically ordered volume, respectively, where the ferromagnetic ground state has a marked $S_{z}$ character yet also displaying finite contributions of $S_{x}$ and $S_{y}$ to the total magnetization. Our results indicate that volume-wise competing electronic phases play an important role in the magnetic properties of $\mathrm{CrI}_{3}$ which set a much lower threshold temperature for exploitation in magnetic device-platforms than initially considered.

Competing electronic phases underlie a number of unusual physical phenomena in condensed matter ${ }^{10}+12$. From superconductivity up to ferromagnetism, when the competition is sizeable the conventional outcome is phase separation. Compounds that have shown such behaviour are mostly of complex magnetic structures including cuprates $\frac{10}{}$, iron-based superconductors ${ }^{12}$, 
ruthenates $s^{11}$, topological kagome magnets ${ }^{3}$ and manganites $s^{13}[14$. A contrasting case is found in the layered transition metal halides ${ }^{15}$ where the presence of heavy halide atoms like in $\mathrm{CrI}_{3}$ stabilises pronounced anisotropy constants resulting in long-range magnetic order in what appears to be a single ferromagnetic transition at a relatively high temperature ${ }^{6 / 16}$. Nevertheless, recent experiments $s^{17-20}$ have unveiled the presence of many subtleties in the magnetism of this compound which a single transition fails to capture. Firstly, $\mathrm{CrI}_{3}$ exhibits both antiferromagnetic and ferromagnetic orders in thin layers driven by hydrostatic pressure ${ }^{17] 18}$. These phases occur at the same critical temperature with a spatial separation of a few hundreds of nanometers and consequently there is no prelude of thermally-activated spin ordering. Secondly, multiple anomalies can be observed in the temperature dependence of the magnetic susceptibility below $\mathrm{T}_{\mathrm{c}}^{9119] 20 \text {. Such }}$ anomalies imply that a more complex magnetic ordering involving spins not directly aligned with the easy-axis can emerge. Whether different magnetic phases may exist or competition occurs between them is largely unknown. However, these observations establish a much more intricate scenario than originally pictured for $\mathrm{CrI}_{3}$ with many hidden features that have important implications in the ordering of the magnetic domains in the system. Here we use high-resolution $\mu \mathrm{SR}$ spectroscopy, complemented by SQUID magnometry and large scale micromagnetic simulations, to systematically study the thermal evolution of magnetic states in $\mathrm{CrI}_{3}$. Such suite is instrumental to identify, characterize and understand distinct macroscopic ground states with any competing magnetic phases.

In a $\mu$ SR experiment, positive muons implanted into a sample serve as extremely sensitive local microscopic probes to detect small internal magnetic fields and ordered magnetic volume 
fractions in the bulk of magnetic systems. See details in Methods and Supplementary Sections S1-S2. Zero-field $\mu$ SR time-spectra are recorded in a powder sample of $\mathrm{CrI}_{3}$ below $(5 \mathrm{~K}, 30 \mathrm{~K}$, $54 \mathrm{~K}$ and $60 \mathrm{~K}$ ) and above $(65 \mathrm{~K}$ and $80 \mathrm{~K}$ ) the magnetic ordering temperature (Fig. 11a-b). A paramagnetic state is generally characterised by a small Gaussian Kubo-Toyabe depolarization of the muon spin originating from the interaction with randomly oriented nuclear magnetic moments. Conversely, the spectra from the highest measured temperature from $150 \mathrm{~K}$ down to $62 \mathrm{~K}$, exhibit a relatively high transverse depolarization rate $\lambda_{T} \simeq 4.9(2) \mu \mathrm{s}^{-1}$. This reflects the occurrence of dense electronic $\mathrm{Cr}$ moments and indicates strong interactions between them. In this scenario a novel correlated paramagnetic state may be present in the system at temperatures above the actual Curie temperature. As the crystal is cooled down, in addition to the paramagnetic signal, an oscillating component with a single well defined frequency is observed at $T \lesssim 62 \mathrm{~K}$ (Fig. 1 1 ab). Below $50 \mathrm{~K}$, a spontaneous muon spin precession with two well-separated distinct precession frequencies is observed in the $\mu \mathrm{SR}$ spectra and persists down to $5 \mathrm{~K}$. The temperature dependences of the internal fields $\left(\mu_{0} H_{\mu}=\omega / \gamma_{\mu}^{-1}\right)$ for the two components are shown in Fig. 2a. The low frequency component shows a monotonous decrease with increasing temperature and disappears at $T_{\mathrm{C} 2} \simeq 50 \mathrm{~K}$. The high frequency component decreases down to $50 \mathrm{~K}$, above which it keeps a constant value within a few Kelvin's range and then decreases again to disappear at $T_{\mathrm{C} 1} \simeq 62 \mathrm{~K}$. Thus, the two oscillatory components have clearly different transition temperatures. This implies the presence of two distinct magnetic transitions in $\mathrm{CrI}_{3}$. We also notice that an upturn on both $\mu_{0} H_{\mu, 1}$ and $\mu_{0} H_{\mu, 2}$ is seen below $T_{\mathrm{C} 3} \simeq 30 \mathrm{~K}$. Moreover, a strongly damped component appears below $T_{\mathrm{C} 3}$ which is seen as some lost of initial asymmetry of the ZF- $\mu$ SR signal. This suggests 
the presence of another magnetic transition at this temperature. The temperature dependences of the relative weights of the individual components in the total $\mu \mathrm{SR}$ signal are shown in Fig. $2 \mathrm{~b}$. The weight of the high frequency component (component I) $\omega_{1}$ gradually increases below $T_{\mathrm{C} 1}$ and reaches maximum at $T_{\mathrm{C} 2}$, below which the second frequency appears. The third component raises below $T_{\mathrm{C} 3} \simeq 30 \mathrm{~K}$. The components I and II share the weight of $(30-70) \%$ in the temperature range between $30 \mathrm{~K}$ and $50 \mathrm{~K}$. These results portray the existence of a clear phase diagram in the temperature domain, in excellent agreement with the magnetic singularities observed by SQUID measurements performed in a single crystal of $\mathrm{CrI}_{3}$ (see Supplementary Figure S2).

Fig. 2c-d show the temperature dependences of the transverse $\lambda_{T}$ and the longitudinal $\lambda_{L}$ depolarisation rates, respectively, of components I and II. The $\lambda_{T}$ is a measure of the width of the static magnetic field distribution at the muon site, and also reflects dynamical effects (spin fluctuations). The $\lambda_{L}$ is determined by dynamic magnetic fluctuations only. For both components, $\lambda_{T}$ is higher than $\lambda_{L}$ in the whole temperature range, indicating that magnetism is mostly static in origin. However, $\lambda_{L 1}$ has a higher overall value than $\lambda_{L 2}$, implying that the magnetic order with $T_{\mathrm{C} 1} \simeq 62 \mathrm{~K}$ contains more dynamics. The presence of three transitions are clearly substantiated by the anomalies, seen in $\lambda_{T}$ and $\lambda_{L}$ (Fig. 2c-d). Namely, the $\lambda_{T, 1}$ starts to increase below $T_{\mathrm{C} 1}$ and peaks at $T_{\mathrm{C} 2}$, then decreases and tends to saturate. Nevertheless, it increments again below $T_{\mathrm{C} 3}$. $\lambda_{T, 2}$ also exhibits an increase below $T_{\mathrm{C} 3}$. Similarly, $\lambda_{L, 1}$ goes to high values for $T<T_{\mathrm{C} 1}$, saturates at $T<T_{\mathrm{C} 2}$ and then enlarges again for $T<T_{\mathrm{C} 3}$, followed by a peak at lower temperature. We note that it is not possible to discriminate in the analysis the contribution of strongly damped components and a high frequency component into $\lambda_{L 1}$ below $30 \mathrm{~K}$ and thus the peak in $\lambda_{L 2}$ at low 
temperatures could be due to the contribution from component III. Overall, these results point to the complex, unconventional thermal evolution of the magnetic states in $\mathrm{CrI}_{3}$.

The behaviour observed involves a volume-wise interplay between various states, providing an important constraint on theoretical models. One possible interpretation of the data is that below $T_{\mathrm{C} 1}$ there is an evolution of the magnetic order in specific volumes of the crystal, which coexists with a correlated paramagnetic state. The second magnetic order thereby occurs within the paramagnetic regions below $T_{\mathrm{C} 2}$. This interpretation is supported by the temperature dependent measurements of the total magnetic fraction $V_{\mathrm{m}}$ (Fig. 3). The magnetic fraction $V_{\mathrm{m}}$ does not acquire the full volume below $T_{\mathrm{C} 1} \simeq 62 \mathrm{~K}$. Instead, it gradually increases below $T_{\mathrm{C} 1}$ and reaches $\simeq$ $80 \%$ at $T_{\mathrm{C} 2} \simeq 50 \mathrm{~K}$. An additional increase of $V_{\mathrm{m}}$ takes place below $T_{\mathrm{C} 3} \simeq 25 \mathrm{~K}$, at which the third strongly damped component appears and reaches nearly $\simeq 100 \%$. The volume wise evolution of magnetic order across $T_{\mathrm{C} 1}, T_{\mathrm{C} 2}$ and $T_{\mathrm{C} 3}$ in $\mathrm{CrI}_{3}$ strongly suggests the presence of distinct magnetic states in the separate volumes of the sample. In addition, we propose that while the frequency of the second component disappears above $50 \mathrm{~K}$, this component exhibits a precursor correlated state up to the highest temperature investigated $150 \mathrm{~K}$.

To understand the microscopic mechanism of these phase transitions, we undertake macroscale spin dynamics which incorporated atomistic (several Ås) and micromagnetic ( $\mu$ m-level) underlying details of the magnetic and electronic structure of $\mathrm{CrI}_{3}$. We modelled the atomistic part using strongly correlated density functional theory based on Hubbard- $U$ methods ${ }^{21}$ whereas the micromagnetic interactions are described through the Landau-Lifshitz-Gilbert (LLG) equation 
techniques 22 . We have also taken into account dipolar fields and higher-order exchange interactions at the level of biquadratic exchange since its sizeable magnitude is important in the magnetic features of $2 \mathrm{D}$ magnets ${ }^{8}$. These approaches have been shown to be critical in the description of the spin properties of halide magnets at high-accuracy of critical temperatures, magnetic domains,

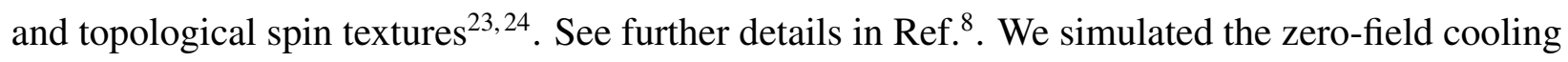
processes for a large square flake of bulk $\mathrm{CrI}_{3}$ of dimensions $0.4 \mu \mathrm{m} \times 0.4 \mu \mathrm{m}$ with a thickness above $200 \mathrm{~nm}$ thickness to avoid any coexistence of ferro- and anti-ferromagnetic phases in the system $^{25}$. As a descriptor of the spin dynamics of the system we utilize the ratio between the number of spins along the $z$-direction or easy-axis $\mathrm{N}\left(\mathrm{S}_{\mathrm{z}}\right)$, relative to the total number $\mathrm{N}\left(\mathrm{S}_{\text {total }}\right)$, that is, $\mathrm{N}\left(\mathrm{S}_{\mathrm{Z}}\right) / \mathrm{N}\left(\mathrm{S}_{\text {total }}\right)$. This allows us to access the number of sites with a specific spin-polarization into the volume of the material which provides information about the disorder caused by thermal fluctuations.

We find that three main phases emerge in $\mathrm{CrI}_{3}$ as function of the temperature, which we name them as Disordered-I, Ordered, and Disordered-II (Figure 4). Each phase is labelled according to the degree of deviation relative to the easy-axis of the magnetization $S_{z}$ (Fig. $4 \mathbf{a}$ ). For instance, the Ordered phase is characteristic of magnitudes of $\left|S_{Z}\right|$ with high spin polarization along $z$, whereas Disordered-II indicates the opposite. The Disordered-I however sets an intermediate phase between both states with different amount of disorder (Fig. 4 b). Surprisingly, the number of spins in the volume of $\mathrm{CrI}_{3}$ with $\left|\mathrm{S}_{\mathrm{Z}}\right|>0.99$, corresponding to the full orientation along the easy-axis, is largely a minority $\left(\mathrm{N}\left(\mathrm{S}_{\mathrm{Z}}\right) / \mathrm{N}\left(\mathrm{S}_{\text {total }}\right) \sim 1.8-3.0 \%\right)$ at the first $\left(\mathrm{T}_{\mathrm{C} 1}=62 \mathrm{~K}\right)$ and second $\left(\mathrm{T}_{\mathrm{C} 2}=50 \mathrm{~K}\right)$ transitions. Most of the sites have a substantial amount of disorder with spins not completely fol- 
lowing the easy-axis of the $\mathrm{CrI}_{3}$ but rather stabilising orientations with in-plane orientations $\left(\mathrm{S}_{\mathrm{X}}\right.$ and $\mathrm{S}_{\mathrm{y}}$ ). Such contributions only become negligible as the temperature drops down below $10 \mathrm{~K}$ where a sharp increment of $\mathrm{N}\left(\mathrm{S}_{\mathrm{Z}}\right) / \mathrm{N}\left(\mathrm{S}_{\text {total }}\right)$ appears which coincides with the antagonistic response of the Disordered-I phase. Spin sites with larger amount of disorder (e.g. Disordered-II) tend to disappear as the system cools down below $40 \mathrm{~K}$. A spatial visualization of the spin orientations, shown in Fig. 4c for different temperatures confirm this description. Remarkably, it shows that mutually exclusive spatial magnetic regions with distinct projection of the magnetization exist in a wide temperature range, which is in perfect agreement with $\mu$ SR results. However, in $\mu$ SR the inhomogeneous and mutually exclusive spatial regions exist even at the base- $T$ of $5 \mathrm{~K}$, while the calculations show a sharp increment of the homogeneous $S_{\mathrm{Z}}$-axis oriented structure below $10 \mathrm{~K}$ (Figure $4 \mathbf{b}$ ). The reason for this discrepancy could be that: (i) there is not a one-to-one correspondence in the temperature values between experiment and theory. (ii) In reality, much lower temperatures than $5 \mathrm{~K}$ are most likely required to get homogeneous magnetic states. It is worth mentioning that even when the system reached $0 \mathrm{~K}$, where thermal fluctuations are inexistent, the spins still evolve in time as an effect due to high magnetic anisotropy and meta-stability of the magnetic domains in $\mathrm{CrI}_{3}{ }^{23}$ (Fig. 4 ). Our results suggest that the magnetic structure of bulk $\mathrm{CrI}_{3}$ is markedly composed by thermal disorder with no apparent formation of anti-ferromagnetic

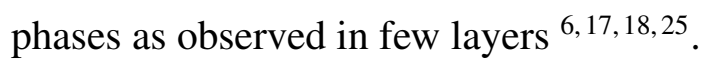




\section{Supplementary Materials}

Materials and Methods.

Supplementary sections S1 to S5, and Figures S1-S2.

\subsubsection{Data Availability}

The data that support the findings of this study are available within the paper and its Supplementary Information.

\subsubsection{Competing interests}

The Authors declare no conflict of interests.

\subsubsection{Acknowledgments}

$\mu \mathrm{SR}$ experiments were performed at at the $\pi \mathrm{M} 3$ beam line (low background GPS instrument) of the Swiss Muon Source (SmuS) of the Paul Scherrer Insitute, Villigen, Switzerland, under proposal ID: 20190297 with EJGS as the PI. GT thank Prof. Alexander Shengelaya and the Georgian National Science Foundation (grant PHDF-19-060) for funding support to participate in $\mu$ SR experiments led by ZG and EJGS. EJGS acknowledges computational resources through the UK Materials and Molecular Modelling Hub for access to THOMAS supercluster, which is partially 
funded by EPSRC (EP/P020194/1); CIRRUS Tier-2 HPC Service (ec131 Cirrus Project) at EPCC (http://www.cirrus.ac.uk) funded by the University of Edinburgh and EPSRC (EP/P020267/1); ARCHER UK National Supercomputing Service (http://www.archer.ac.uk) via Project d429. EJGS acknowledges the EPSRC Early Career Fellowship (EP/T021578/1) and the University of Edinburgh for funding support. ENM acknowledges the European Research Council (ERC) under the Horizon 2020 research and innovation programme (ERC StG, grant agreement No. 803092).

\section{References and Notes}

1. Luetkens, H. et al. Microscopic evidence of spin state order and spin state phase separation in layered cobaltites $r_{b^{2}} \mathrm{Oaco}_{2.5}$ with $r=\mathrm{y}$, tb, dy, and ho. Phys. Rev. Lett. 101, 017601 (2008). URL https://link.aps.org/doi/10.1103/PhysRevLett.101.017601.

2. Hiraishi, M. et al. Bipartite magnetic parent phases in the iron oxypnictide superconductor. Nature Physics 10, 300-303 (2014). URL https://doi.org/10.1038/nphys2906.

3. Guguchia, Z. et al. Tunable anomalous hall conductivity through volume-wise magnetic competition in a topological kagome magnet. Nature Communications 11, 559 (2020). URL https://doi.org/10.1038/s41467-020-14325-w.

4. Guguchia, Z. et al. Magnetism in semiconducting molybdenum dichalcogenides. Science Advances 4 (2018). URL https://advances.sciencemag.org/content/4/12/eaat3672. https://advances.sciencemag.org/content/4/12/eaat3672.full.pdf. 
5. Niedermayer, C. et al. Common phase diagram for antiferromagnetism in $\mathrm{la}_{2-x} \mathrm{sr}_{x} \mathrm{CuO}_{4}$ and $Y_{1-x} \mathrm{ca}_{x} \mathrm{ba}_{2} \mathrm{cu}_{3} O_{6}$ as seen by muon spin rotation. Phys. Rev. Lett. 80, 3843-3846 (1998). URL https://link.aps.org/doi/10.1103/PhysRevLett.80.3843.

6. Huang, B. et al. Layer-dependent ferromagnetism in a van der waals crystal down to the monolayer limit. Nature 546, 270-273 (2017).

7. Gong, C. et al. Discovery of intrinsic ferromagnetism in two-dimensional van der waals crystals. Nature 546, 265-269 (2017). URL http://dx.doi.org/10.1038/nature22060. Letter.

8. Kartsev, A., Augustin, M., Evans, R. F. L., Novoselov, K. S. \& Santos, E. J. G. Higher-order exchange interactions in two-dimensional magnets. arXiv:2006.04891 (2020).

9. McGuire, M. a., Dixit, H., Cooper, V. R. \& Sales, B. C. Coupling of crystal structure and magnetism in the layered, ferromagnetic insulator CrI3. Chem. Mater. 27, 612-620 (2015).

10. Mohottala, H. E. et al. Phase separation in superoxygenated la2-xsrxcuo4+y. Nature Materials 5, 377-382 (2006). URL https://doi.org/10.1038/nmat1633.

11. Uemura, Y. J. et al. Phase separation and suppression of critical dynamics at quantum phase transitions of mnsi and (sr1-xcax)ruo3. Nature Physics 3, 29-35 (2007). URL https://doi. org/10.1038/nphys488.

12. de la Cruz, C. et al. Magnetic order close to superconductivity in the iron-based layered lao1-xf x feas systems. Nature 453, 899-902 (2008). URL https://doi.org/10.1038/ nature07057. 
13. Milward, G. C., Calderón, M. J. \& Littlewood, P. B. Electronically soft phases in manganites. Nature 433, 607-610 (2005). URL https ://doi .org/10 .1038/nature03300.

14. Uehara, M., Mori, S., Chen, C. H. \& Cheong, S. W. Percolative phase separation underlies colossal magnetoresistance in mixed-valent manganites. Nature 399, 560-563 (1999). URL https://doi.org/10.1038/21142.

15. de Jongh, L. Magnetic Properties of Layered Transition Metal Compounds (Springer, 2012).

16. Dillon, J. F. \& Olson, C. E. Magnetization, resonance, and optical properties of the ferromagnet CrI3. J. Appl. Phys. 36, 1259-1260 (1965).

17. Song, T. et al. Switching $2 \mathrm{~d}$ magnetic states via pressure tuning of layer stacking. Nature Materials 18, 1298-1302 (2019). URL https://doi.org/10.1038/s41563-019-0505-2.

18. Li, T. et al. Pressure-controlled interlayer magnetism in atomically thin cri3. Nature Materials 18, 1303-1308 (2019). URL https://doi.org/10.1038/s41563-019-0506-1.

19. Liu, Y. \& Petrovic, C. Anisotropic magnetocaloric effect in single crystals of CrI3. Phys. Rev. B Condens. Matter 97, 174418 (2018).

20. Wang, Z. et al. Very large tunneling magnetoresistance in layered magnetic semiconductor CrI3. Nat. Commun. 9, 2516 (2018).

21. Dudarev, S. L., Botton, G. A., Savrasov, S. Y., Humphreys, C. J. \& Sutton, A. P. Electronenergy-loss spectra and the structural stability of nickel oxide: An lsda+u study. Phys. Rev. B 57, 1505-1509 (1998). URL https://link.aps.org/doi/10.1103/PhysRevB.57.1505. 
22. Nakatani, Y., Uesaka, Y. \& Hayashi, N. Direct solution of the landau-lifshitz-gilbert equation for micromagnetics. Japanese Journal of Applied Physics 28, 2485-2507 (1989). URL https://doi.org/10.1143\%2Fjjap.28.2485.

23. Wahab, D. A. et al. Quantum rescaling, metastability and hybrid domain-walls in twodimensional cri3 magnets. arXiv: (2020).

24. Augustin, M., Jenkins, S., Evans, R. F. L., Novoselov, K. S. \& Santos, E. J. G. Properties and dynamics of meron topological spin textures in the two-dimensional magnet $\mathrm{crcl} 3$. arXiv: (2020).

25. Niu, B. et al. Coexistence of magnetic orders in two-dimensional magnet cri3. Nano Letters 20, 553-558 (2020). URL https://doi.org/10.1021/acs.nanolett.9b04282. 


\section{Figure captions}

Figure 1: $\mu$ SR spectroscopy applied to $\mathbf{C r I}_{3}$. a-b, Zero-field $\mu$ SR spectra, recorded at various temperatures for the polycrystalline sample of $\mathrm{CrI}_{3}$, shown in the low and extended time interval. The solid lines are the fit of the data using the methods of Supplementary Sections S3-S4. Error bars are the standard error of the mean in about $10^{6}$ events. The error of each bin count is given by the standard deviation of $n$. The errors of each bin in the $\mu$ SR asymmetry are then calculated by statistical error propagation.

Figure 2: Temperature dependent $\mu$ SR parameters. a, The temperature dependence of the internal magnetic fields for the observed two components in $\mathrm{CrI}_{3}$. b, The temperature dependence of the relative weights of the three components in the total signal for $\mathrm{CrI}_{3}$, determined from zero-field $\mu \mathrm{SR}$ experiments. c-d, The temperature dependence of transverse depolarization rates $\lambda_{T 1}, \lambda_{T 2}$ and the longitudinal depolarization rates $\lambda_{L 1}, \lambda_{L 2}$ for two components. The error bars represent the standard deviation of the fit parameters.

Figure 3: Thermal evolution of various magnetic phases in $\mathbf{C r I}_{3}$. a, The temperature dependence of the total magnetic volume fraction $V_{M}$, determined from precise weak transverse field (weak-TF) $\mu$ SR measurements. In this weak-TF experiment, a small magnetic field of $30 \mathrm{G}$ is applied nearly perpendicular to the muon spin polarisation. The different components seen in Fig. 2 are highlighted in each region of the temperature range with the paramagnetic phase above the Curie temperature. The error bars represent the standard deviation of the fit parameters. 
Figure 4: Micromagnetic analysis of the spin dynamics in $\mathbf{C r I}_{3}$. a, Diagram of the different magnitudes of $\left|S_{z}\right|$ relative to the unit sphere in the range of $\left|S_{z}\right|=1$ (full polarized along $z$ ) and $\left|S_{z}\right|=0$ (only in-plane $x y$-projections). The orientations of the spins are assigned randomly following the Monte Carlo moves. The plot only shows a schematic of possible projections over the sphere with others (not shown) with the same $\left|S_{z}\right|$ equally probable. b, Magnetic fraction (in \%) of the out-of-plane spins $\left(\mathrm{N}\left(\mathrm{S}_{\mathrm{Z}}\right) / \mathrm{N}\left(\mathrm{S}_{\text {total }}\right)\right)$ as a function of the temperature. The calculation of $\mathrm{N}\left(\mathrm{S}_{\mathrm{z}}\right)$ and $\mathrm{N}\left(\mathrm{S}_{\text {total }}\right)$ take into account the volumetric amount of spins over the entire system for $\mathrm{S}_{\mathrm{Z}}$ and $\mathrm{S}_{\text {total }}$, respectively. Three main phases were identified in bulk $\mathrm{CrI}_{3}$ which are named: Disordered-I (faint black), with values of $\mathrm{N}\left(\mathrm{S}_{\mathrm{Z}}\right) / \mathrm{N}\left(\mathrm{S}_{\text {total }}\right)$ between the curves of $0.01<\left|S_{z}\right|<0.99$ and $0.20<\left|S_{z}\right|<0.80$; Ordered (faint red), within $\left|S_{z}\right|>0.80$ and $\left|S_{z}\right|>$ 0.99; and, Disordered-II (faint blue), within $\left|S_{z}\right|<0.01$ and $\left|S_{z}\right|<0.20$. The solid curves showed in each phase provide a sample of a specific variation of $\mathrm{N}\left(\mathrm{S}_{\mathrm{z}}\right) / \mathrm{N}\left(\mathrm{S}_{\text {total }}\right)$ for a given range of $\left|\mathrm{S}_{\mathrm{Z}}\right|$. The vertical dashed line sets the time of $2 \mathrm{~ns}$ required to achieve $0 \mathrm{~K}$ in the spin dynamics. The magnitudes of $\mathrm{N}\left(\mathrm{S}_{\mathrm{Z}}\right) / \mathrm{N}\left(\mathrm{S}_{\text {total }}\right)$ showed after $0 \mathrm{~K}$ demonstrated that even when the thermal fluctuations are zero, the system is still evolving to stabilize its ground state. The critical temperatures $\left(\mathrm{T}_{\mathrm{C} 1, \mathrm{C} 2, \mathrm{C} 3}\right)$ are also highlighted. $\mathbf{c}$, Snapshots of the dynamical spin configurations of bulk $\mathrm{CrI}_{3}$ during field cooling from $80 \mathrm{~K}$ down to $0 \mathrm{~K}$. Magnetisation is projected along of in-plane components $\left(\mathrm{S}_{\mathrm{x}}, \mathrm{S}_{\mathrm{y}}\right)$ and $S_{Z}$ at different temperatures: $68 \mathrm{~K}, 60 \mathrm{~K}, 50 \mathrm{~K}, 20 \mathrm{~K}$ and $0 \mathrm{~K}$. Each column and row corresponds to a specific projection of the magnetization at a given temperature provided at the far left. The colour scale shows the variation of the three magnetization components $\left(S_{x}, S_{y}, S_{z}\right)$ throughout the system. 


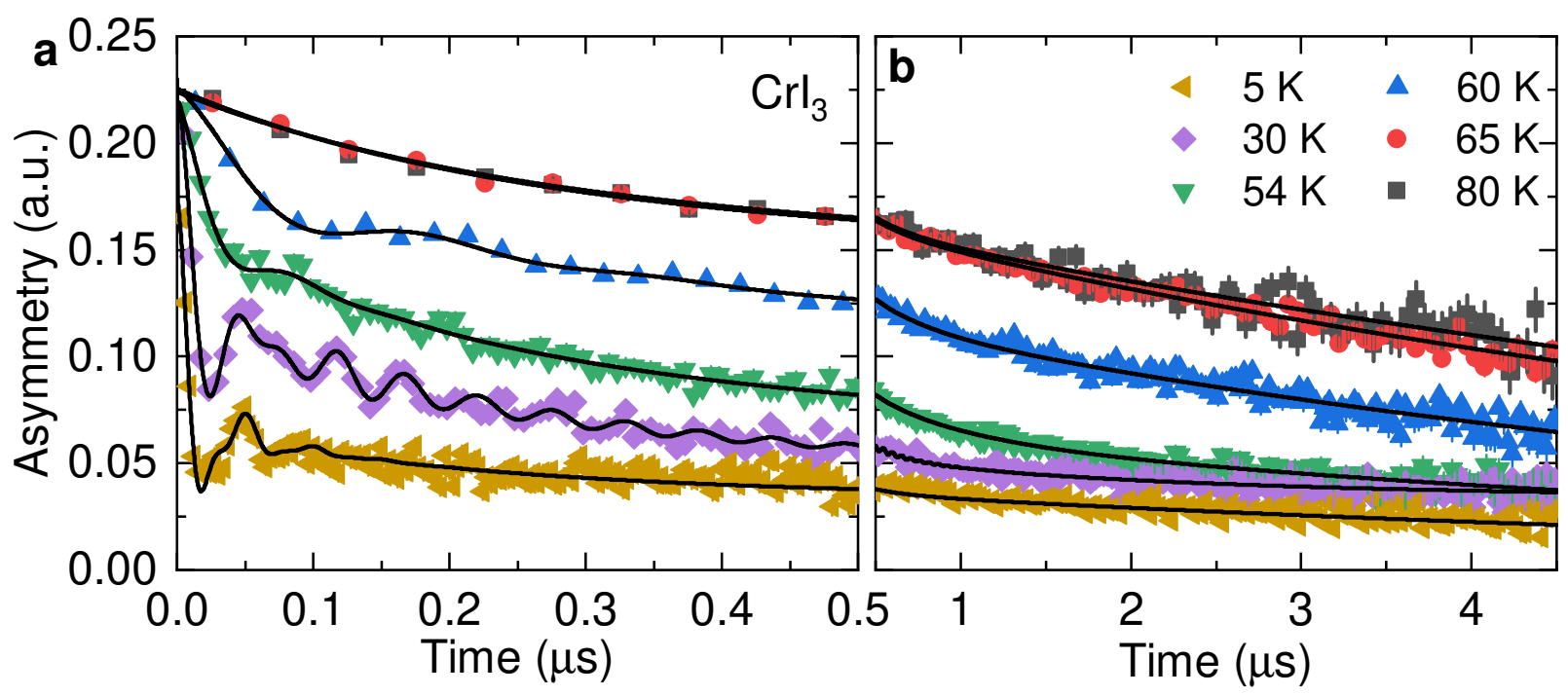

Figure 1 

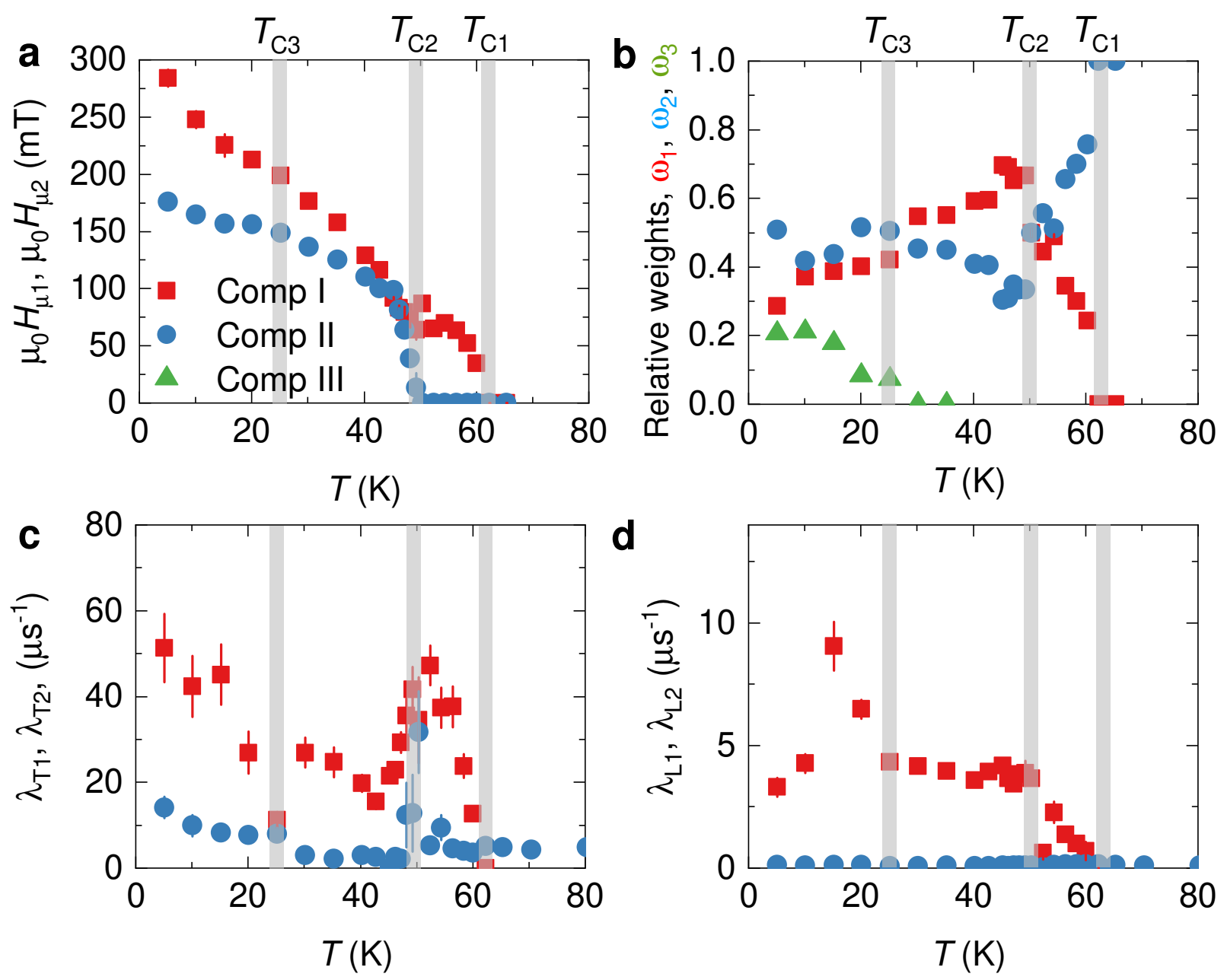

Figure 2 


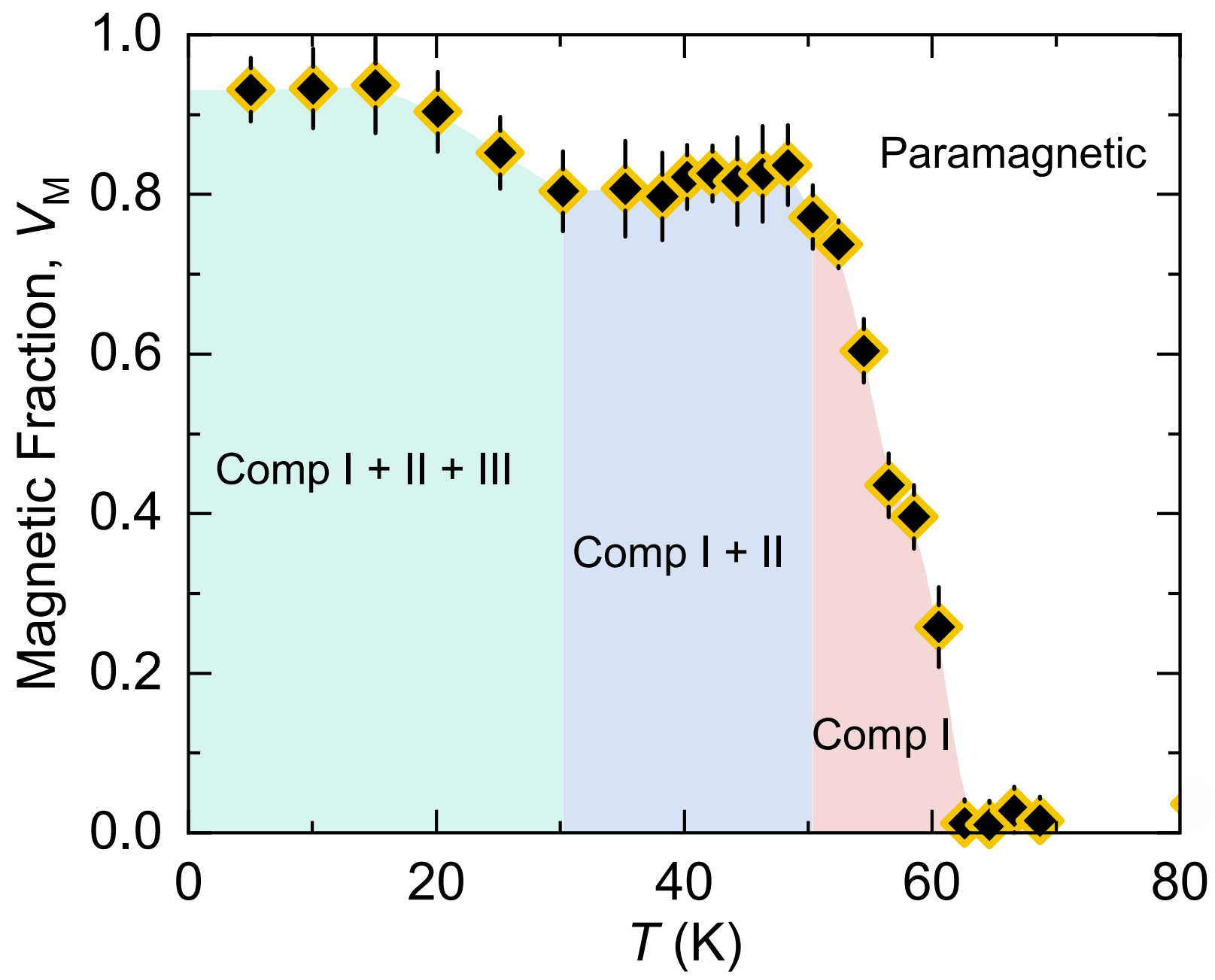

Figure 3 
a

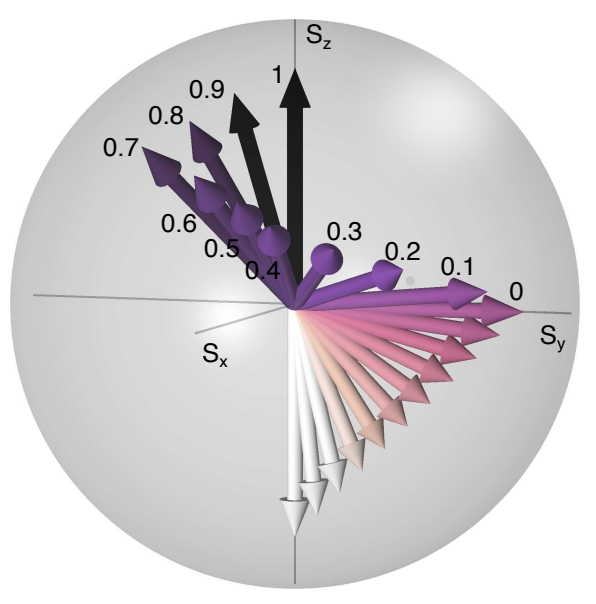

C
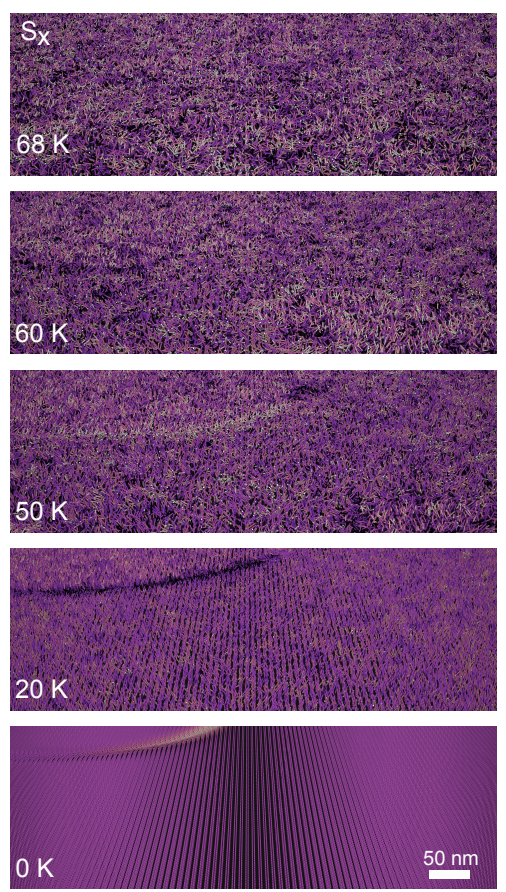
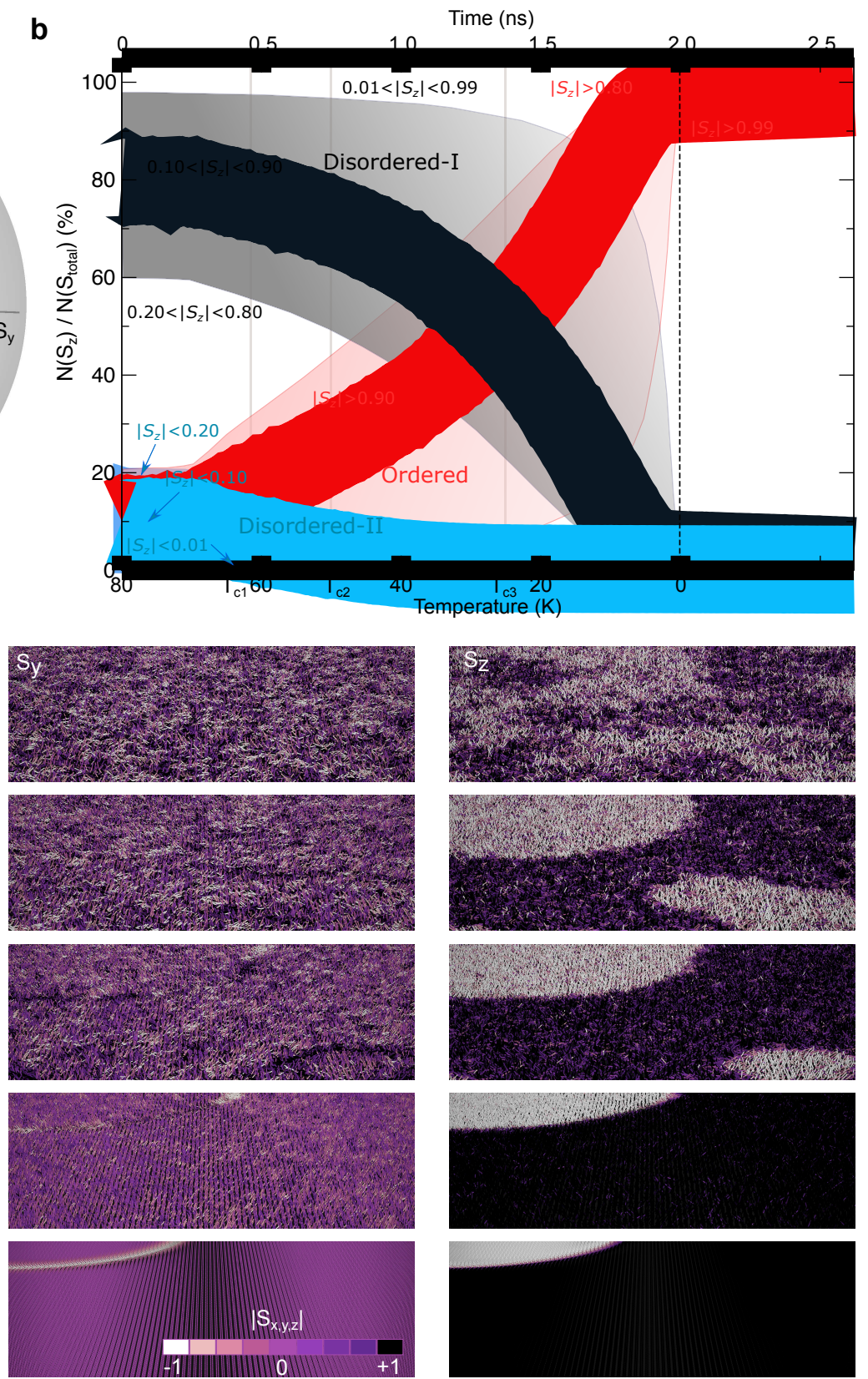
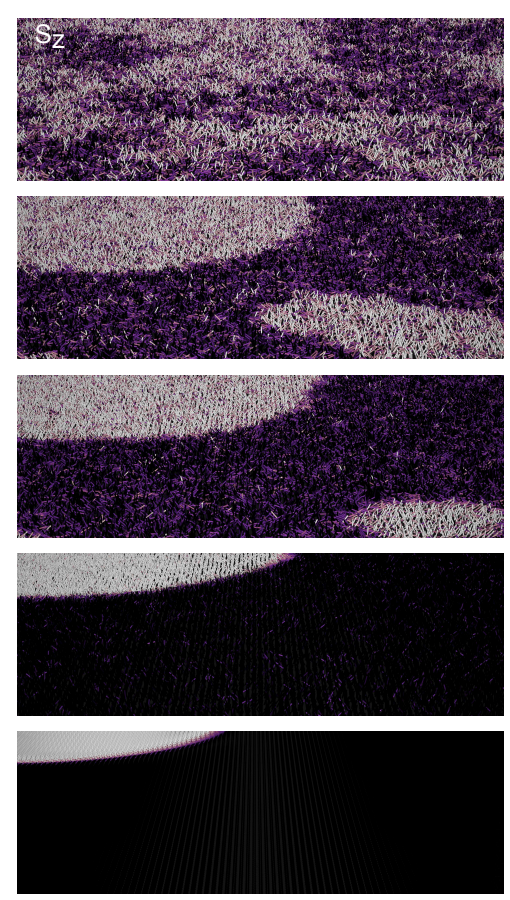


\section{Thermal disorder driven magnetic phases in van der Waals magnet $\mathrm{Crl}_{3}$}

4 Jaume Meseguer-Sánchez ${ }^{1}$, Dina Abdul Wahab ${ }^{2}$, Hubertus Luetkens ${ }^{3}$, Grigol Taniashvili ${ }^{4}$, Efrén

${ }_{5}$ Navarro-Moratalla ${ }^{1, \dagger}$, Zurab Guguchia ${ }^{3, \dagger}$, Elton J. G. Santos ${ }^{5, \dagger}$

${ }_{6}{ }^{1}$ Instituto de Ciencia Molecular, Universitat de Valéncia, Calle Catedrático José Beltrán Martínez

7 2, 46980, Paterna, Spain

${ }_{8}^{2}$ School of Mathematics and Physics, Queen's University Belfast, BT7 1NN, United Kingdom

$9{ }^{3}$ Laboratory for Muon Spin Spectroscopy, Paul Scherrer Institute, CH-5232 Villigen PSI, Switzer-

10 land

${ }_{11}^{4}$ Department of Physics, Tbilisi State University, Chavchavadze 3, GE-0128 Tbilisi, Georgia

${ }_{12}^{5}$ Institute for Condensed Matter Physics and Complex Systems, School of Physics and Astronomy,

13 The University of Edinburgh, EH9 3FD, UK

${ }_{14}^{\dagger}$ Correspondences to: efren.navarro@me.com, zurab.guguchia@psi.ch, esantos@ed.ac.uk

\section{Contents}

16

$1 \mathrm{CrI}_{3}$ bulk crystal growth 


\section{$1 \mathrm{CrI}_{3}$ bulk crystal growth}

Chromium triiodide crystals were grown using the chemical vapour transport technique. Chromium powder (99.5\% Sigma-Aldrich) and anhydrous iodine beads (99.999\% Sigma-Aldrich) were mixed in a 1:3 ratio in an argon atmosphere inside a glovebox. $972 \mathrm{mg}$ of the mixture were loaded into a silica ampoule with a length, inner diameter and outer diameter of $500 \mathrm{~mm}, 15 \mathrm{~mm}$ and $16 \mathrm{~mm}$ respectively (Supplementary Figure S1a). The ampoule was extracted from the glovebox with a ball valve covering the open end to prevent air exposure and then it was immediately was evacuated using a turbo-molecular pump down to $6 \times 10^{-6}$. Once the pressure stabilised, the closed end was dipped in liquid nitrogen to prevent the sublimation of the iodide beads. The ampoule was then flame sealed and introduced into a three-zone furnace. An initial inverted gradient step was used to minimize nucleation sites in the growth zone. The gradient was then inverted so that the source zone would remain at $650{ }^{\circ} \mathrm{C}$, the middle growth zone at $550{ }^{\circ} \mathrm{C}$ and the third zone at $600{ }^{\circ} \mathrm{C}$ for 3 days. Crystal growth takes place in the centre and source zones. The single crystals and the microcrystalline powder were both extracted from the ampoule and stored in an argon atmosphere inside the glovebox to prevent oxidation and hydration. A sample of the bigger single crystals was 
a

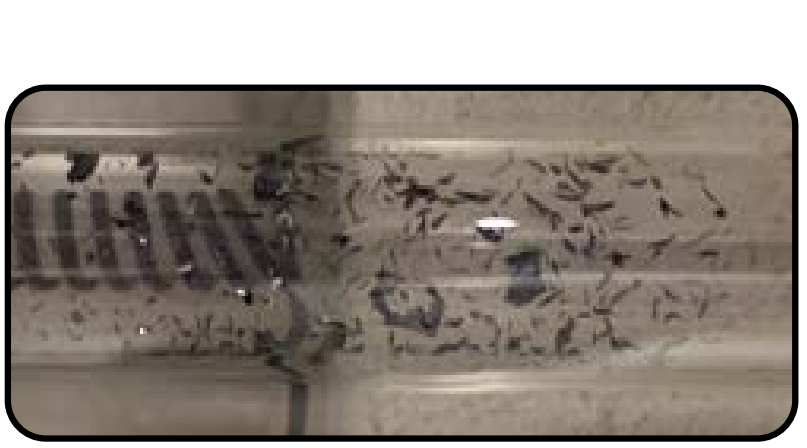

b

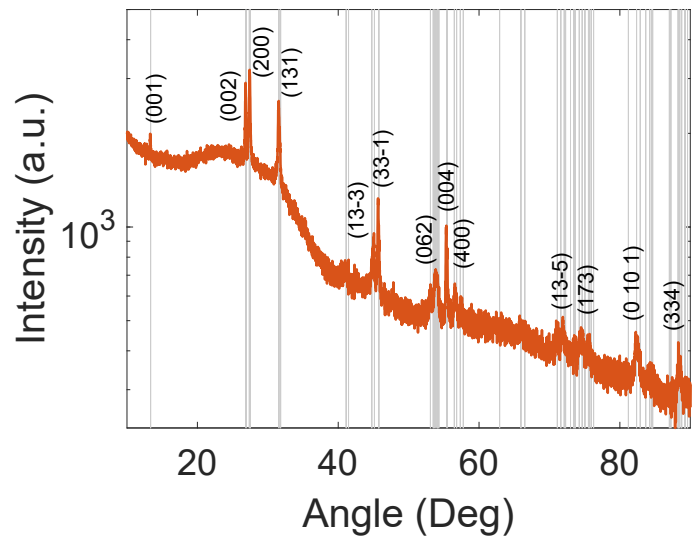

Figure S1: a, Picture of the ampoule used for sample fabrication. b, X-Ray diffraction pattern of the $\mathrm{CrI}_{3}$ microcrystalline powder studied in the $\mu$ SR experiment. The most prominent peaks of the orange trace (experiment) have been labelled (Miller indices) and marked (grey lines) with peaks positions of the monoclinic (room temperature) crystalline phase of $\mathrm{CrI}_{3}$. 


\section{$2 \quad \mu \mathrm{SR}$ experiment}

The $\mu$ SR method is based on the observation of the time evolution of the spin polarization $\vec{P}(\mathrm{t})$ of the muon ensemble. In a $\mu \mathrm{SR}$ experiments an intense beam $\left(p_{\mu}=29 \mathrm{MeV} / \mathrm{c}\right)$ of $100 \%$ spinpolarized muons is stopped in the sample. Currently available instruments allow essentially a background free $\mu$ SR measurement at ambient conditions ${ }^{1}$. The positively charged muons thermalize in the sample at interstitial lattice sites, where they act as magnetic microprobes. In a magnetic material the muons spin precess in the local field $B_{\mu}$ at the muon site with the Larmor frequency $v_{\mu}=\gamma_{\mu} /(2 \pi) B_{\mu}$ (muon gyromagnetic ratio $\gamma_{\mu} /(2 \pi)=135.5 \mathrm{MHz} \mathrm{T}^{-1}$ ). The muons $\mu^{+}$implanted into the sample will decay after a mean life time of $\tau_{\mu}=2.2 \mu \mathrm{s}$, emitting a fast positron $e^{+}$preferentially along their spin direction. Various detectors placed around the sample track the incoming $\mu^{+}$and the outgoing $e^{+}$. When the muon detector records the arrival of a $\mu$ in the specimen, the electronic clock starts. The clock is stopped when the decay positron $e^{+}$is registered in one of the $e^{+}$detectors, and the measured time interval is stored in a histogramming memory. In this way a positron-count versus time histogram is formed. A muon decay event requires that within a certain time interval after a $\mu^{+}$has stopped in the sample a $e^{+}$is detected. This time interval extends usually over several muon lifetimes (e.g. 10 $\mu \mathrm{s})$.

The GPS ( $\pi \mathrm{M} 3$ beamline) $\mu \mathrm{SR}$ instrument at the Paul Scherrer Institute, Switzerland, was used to study the $\mathrm{CrI}_{3}$ sample. The specimen was mounted in a He gas-flow cryostat and the temperature was varied between 5 and $200 \mathrm{~K}$. 


\section{Analysis of Weak TF- $\mu$ SR data}

62 The weak TF asymmetry spectra were analyzed ${ }^{2,3}$ using the function:

$$
A_{S}(t)=A_{p} \exp (-\lambda t) \cos (\omega t+\phi)
$$

where $t$ is the time after muon implantation, $A(t)$ is the time-dependent asymmetry, $A_{p}$ is the amplitude of the oscillating component (related to the paramagnetic volume fraction), $\lambda$ is an exponential damping rate due to paramagnetic spin fluctuations and/or nuclear dipolar moments, $\omega=2 \pi v_{\mu}$ is the Larmor precession frequency set by the strength of the transverse magnetic field, and $\phi$ is a phase offset. The zero for $A(t)$ was allowed to vary for each temperature to deal with the asymmetry baseline shift occurring for magnetically ordered samples. From these refinements, the magnetically ordered volume fraction at each temperature $T$ was obtained by $V_{M}=1-A_{p}(T) / A_{p}\left(T_{\max }\right)$, where $A_{p}\left(T_{\max }\right)$ is the amplitude in the paramagnetic phase at high temperature. 


\section{Analysis of $\mathrm{ZF}-\mu \mathrm{SR}$ data}

In the paramagnetic state, the $\mu \mathrm{SR}$ spectra are described by the combination of Lorentzian and Gaussian Kubo-Toyabe depolarization function:

$$
A_{\mathrm{ZF}, \mathrm{PM}}(t)=\left(1-V_{M}\right) A_{0}\left(\frac{1}{3}+\frac{2}{3}\left(1-(\sigma t)^{2}-\Lambda t\right) e^{-\frac{1}{2}(\sigma t)^{2}-\Lambda t}\right)
$$

Here, the depolarisation rates $\sigma$ and $\Lambda$ are due to the nuclear dipole moments and randomly oriented diluted local electronic moments, respectively. $A_{0}$ is the initial asymmetry. $V_{P M}=1-V_{M}$ is the paramagnetic fraction, which acquires maximum value of 1 above $60 \mathrm{~K}$.

In the ordered state, the response of the sample consists of three magnetic components, depending on temperature:

$$
\begin{aligned}
A_{\mathrm{ZF}, \mathrm{M}}(t)= & V_{M} A_{0} \omega_{1}\left(f_{T 1} \cos \left(\gamma_{\mu} B_{\mu 1} t+\phi\right) e^{-\lambda_{T 1} t}+f_{L 1} e^{-\lambda_{L 1} t}\right)+\cdots \\
& V_{M} A_{0} \omega_{2}\left(f_{T 2} \cos \left(\gamma_{\mu} B_{\mu 2} t+\phi\right) e^{-\lambda_{T 2} t}+f_{L 2} e^{-\lambda_{L 2} t}\right)+\cdots \\
& V_{M} A_{0} \omega_{3}\left(f_{T 3} e^{-\lambda_{T 3} t}+f_{L 3} e^{-\lambda_{L 3} t}\right)
\end{aligned}
$$

Here, $\omega_{1}, \omega_{2}$, and $\omega_{3}$ are the relative fractions of the magnetic components. The first and second terms describe the high and low frequency magnetic components, respectively. The components are characterised by an oscillating "transverse" component and a slowly relaxing "lon- 
gitudinal" component. The longitudinal component arises due to muons experiencing local field components which are parallel to the initial muon spin polarization. In polycrystalline samples with randomly oriented fields the orientational averaging results in a so-called "one-third tail" with $f_{L}=\frac{1}{3}\left(f_{T}=\frac{2}{3}\right)$. For single crystals, $f_{L}$ varies between zero and unity as the orientation between field and polarization changes from being perpendicular to parallel. $\lambda_{T}$ and $\lambda_{L}$ are the transverse and longitudinal depolarisation rates of the $\mu$ SR signals, respectively. $B_{\mu 1}$ and $B_{\mu 2}$ are the internal magnetic fields at the muon site, corresponding to high and low frequency components, respectively. The third term describes the strongly damped magnetic component, which occurs below 30 K only. We note that it is not possible to separate $\lambda_{L 3}$ from $\lambda_{L 1}$ and $\lambda_{L 2}$ and thus it is fixed to 0 in the analysis. 


\section{SQUID magnetometry}

Sample preparation In order to measure the magnetization of $\mathrm{CrI}_{3}$ in the presence of in-plane and out-of-plane external magnetic fields, two different samples were prepared. For the out-of-plane orientation, various single crystals were stacked one on top of the other and placed onto the surface on the end of a polyethylene rod. This was carefully placed inside a silica tube with the crystals facing upwards and another polyethylene rod placed on top trapping the crystal in between. For the in-plane orientation, a polyethylene rod was cut longitudinally and a single crystal was glued to the flat using Apiezon-N grease. These preparations were performed in an argon atmosphere to avoid crystal degradation. The samples were then inserted inside an outer silica tube and flame sealed at a pressure lower than $10^{-3}$ mbar.

Magnetization and magnetic susceptibility of $\mathrm{CrI}_{3}$ crystals Magnetization curves and zerofield-cooled/field-cooled susceptibility sweeps were carried out in a SQUID magnetomoter (Quantum Design MPMS-XL-7) on single crystals of $\mathrm{CrI}_{3}$ were the relative orientation of the basal plane of the sample with the external magnetic field (both AC and DC) is controlled. We find signatures of three independent magnetic phase transitions consistent with the $\mu$ SR experiments (Supplementary Fig.S2). The most prominent feature in the thermal variation of the magnetic susceptibility onsets at $61 \mathrm{~K}$ as shown by the DC measurements reflected in Supplementary Fig. S2a-b, in good agreement with recent experiments performed on this material ${ }^{4}$. The real part of the AC measurements shows that this transition independent of the AC drive frequency (Supplementary Fig.S2c-d). Interestingly, there is no peak in the imaginary component of the AC magnetization 
in both orientations. A second feature at $50 \mathrm{~K}$ is particularly visible in the thermal dependence of the in-plane magnetic moment of the crystal shown in Supplementary Fig. S2b, d. This can be attributed to a second magnetic phase transition as discussed in the main text. The presence of this additional peak was mentioned in previous experiments ${ }^{5}$ however without any explanation or characterization. As with the main critical feature, this peak also exhibits no frequency dependence, which suggests that this phase transition is a long-range ordering. The $50 \mathrm{~K}$ maximum is most prominent for the in-plane orientation measurements (both AC and DC), suggesting that some component of the magnetization exists in the crystallographic $a b$-plane. This observation complements the notion of a strong magnetic anisotropy along the $c$-axis and, at the same time, reveals the presence of some in-plane magnetic moment. Finally, the fact that the imaginary component of the AC magnetisation for the in-plane orientation in Supplementary Fig. S2c exhibits an onset around $30 \mathrm{~K}$ marks the onset of the third phase transition. 
a

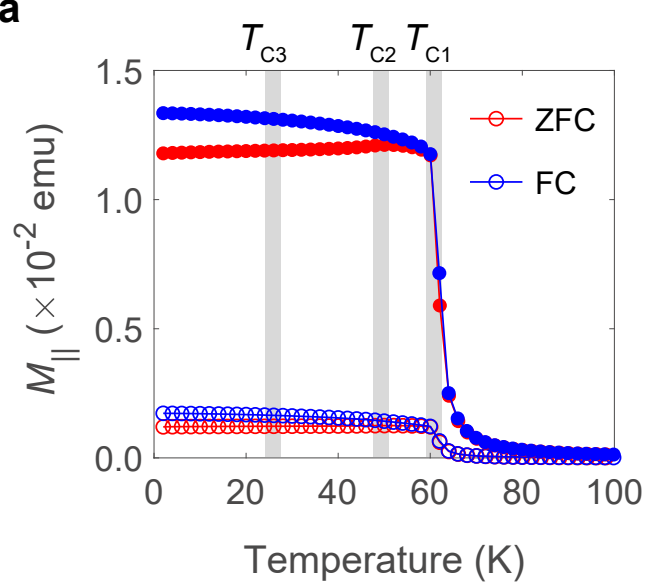

C

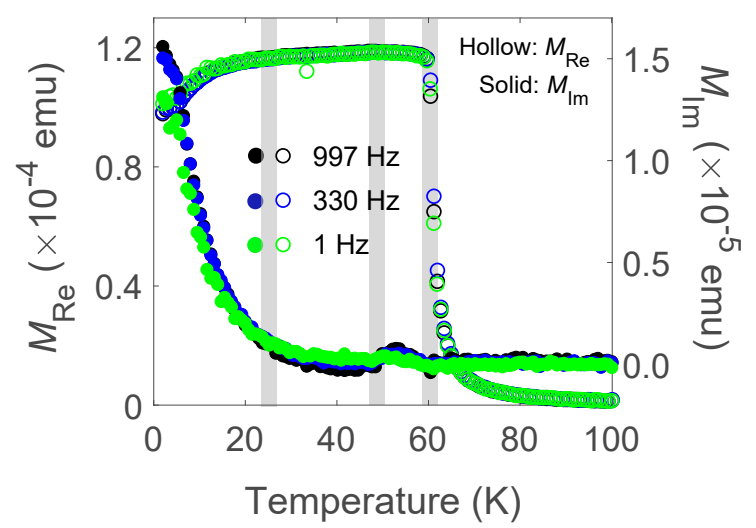

b

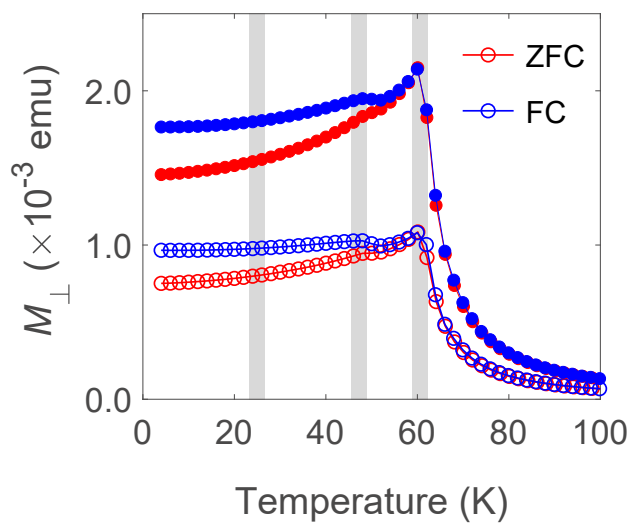

d

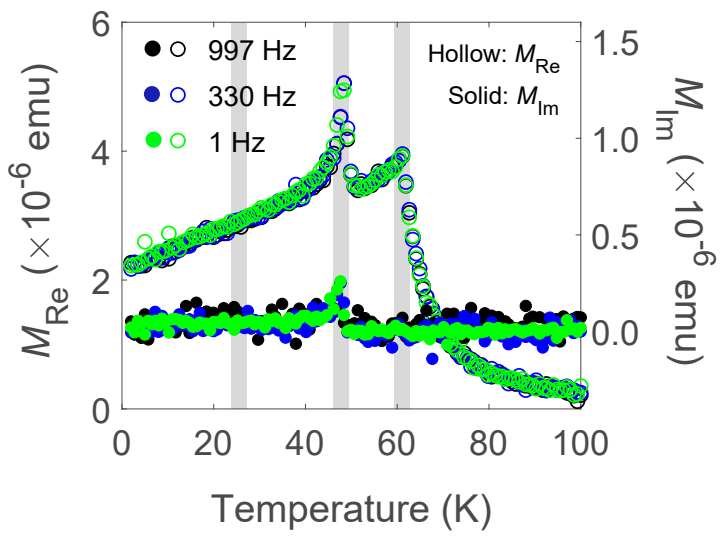

Figure S2: a-b, Zero-field-cooled/field-cooled temperature dependence of the DC magnetization at different external magnetic fields, aligned parallel and perpendicular to the crystallographic c-axis respectively. The DC external magnetic field used was $10 \mathrm{G}$ in a and $50 \mathrm{G}$ in $\mathbf{b}$, represented by the empty markers. The solid markers represent the data measured with an external field of $100 \mathrm{G}$ for both orientations. The solid line is a guide to the eye. c-d, Zerofield temperature dependence of the $\mathrm{AC}$ magnetization at three different frequencies for the parallel and perpendicular orientations, respectively. The real and imaginary components are represented by the hollow and solid markers, respectively. The grey-shaded regions are a reference for the reader indicating the temperatures at which the magnetic anomalies are seen. The regions are consistent with those shaded in Figure 2 for comparison. 


\section{Supplementary References}

1. Amato, A. et al. The new versatile general purpose surface muon instrument. Review of Scientific Instruments 88, 093301 (2017). URL https://doi.org/10.1063/1.4986045.

2. Suter, A. \& Wojek, B. Musrfit: A free platform-independent framework for musr data analysis. Physics Procedia 30, 69 - 73 (2012). URL http://www.sciencedirect.com/science/ article/pii/S187538921201228X.

3. Guguchia, Z. et al. Tunable anomalous hall conductivity through volume-wise magnetic competition in a topological kagome magnet. Nature Communications 11, 559 (2020). URL https://doi.org/10.1038/s41467-020-14325-w.

4. de la Cruz, C. et al. Magnetic order close to superconductivity in the iron-based layered lao1-xf x feas systems. Nature 453, 899-902 (2008). URL https://doi.org/10.1038/ nature07057.

5. McGuire, M. a., Dixit, H., Cooper, V. R. \& Sales, B. C. Coupling of crystal structure and magnetism in the layered, ferromagnetic insulator CrI3. Chem. Mater. 27, 612-620 (2015). 


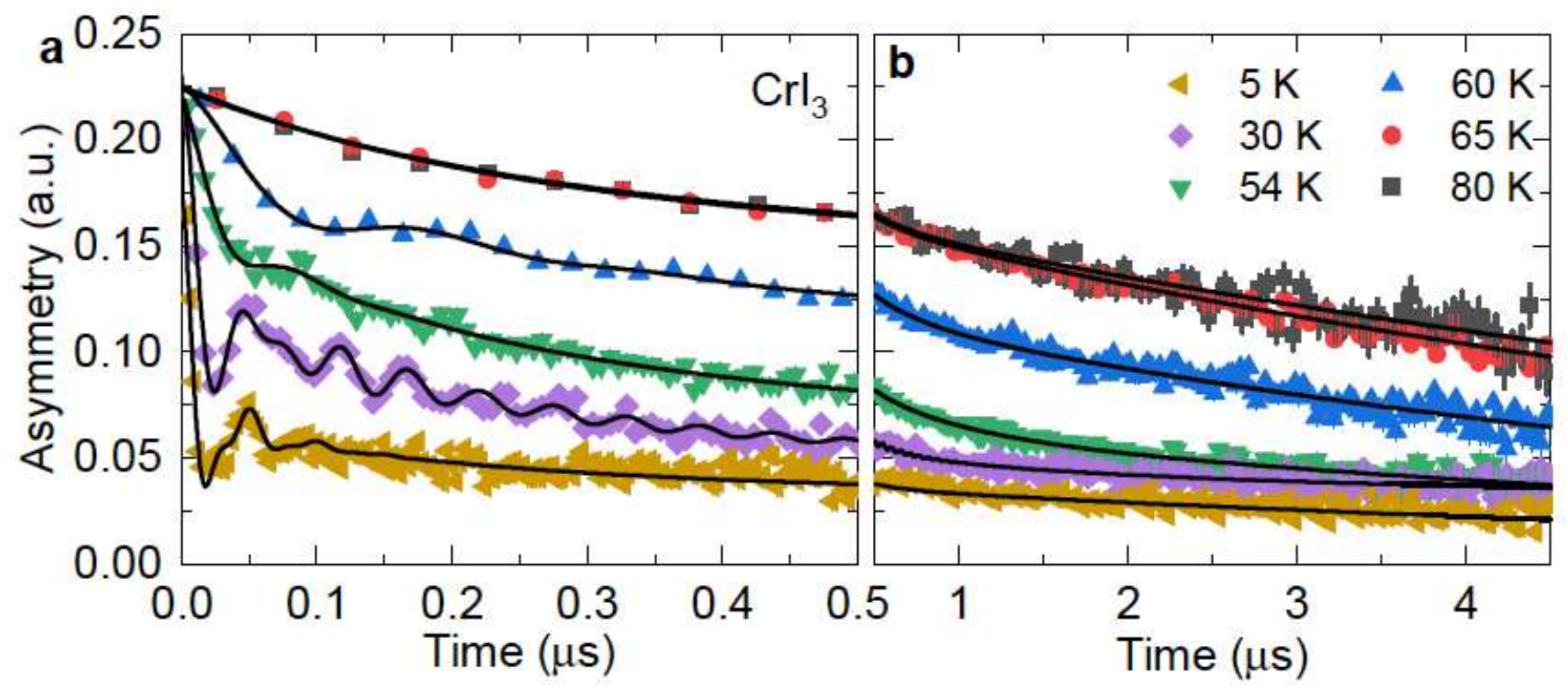

Figure 1

\section{Figure 1}

$\mu S R$ spectroscopy applied to Crl3. a-b, Zero-field $\mu S R$ spectra, recorded at various temperatures for the polycrystalline sample of Crl3, shown in the low and extended time interval. The solid lines are the fit of the data using the methods of Supplementary Sections S3-S4. Error bars are the standard error of the mean in about 106 events. The error of each bin count is given by the standard deviation of $\mathrm{n}$. The errors of each bin in the $\mu \mathrm{SR}$ asymmetry are then calculated by statistical error propagation. 

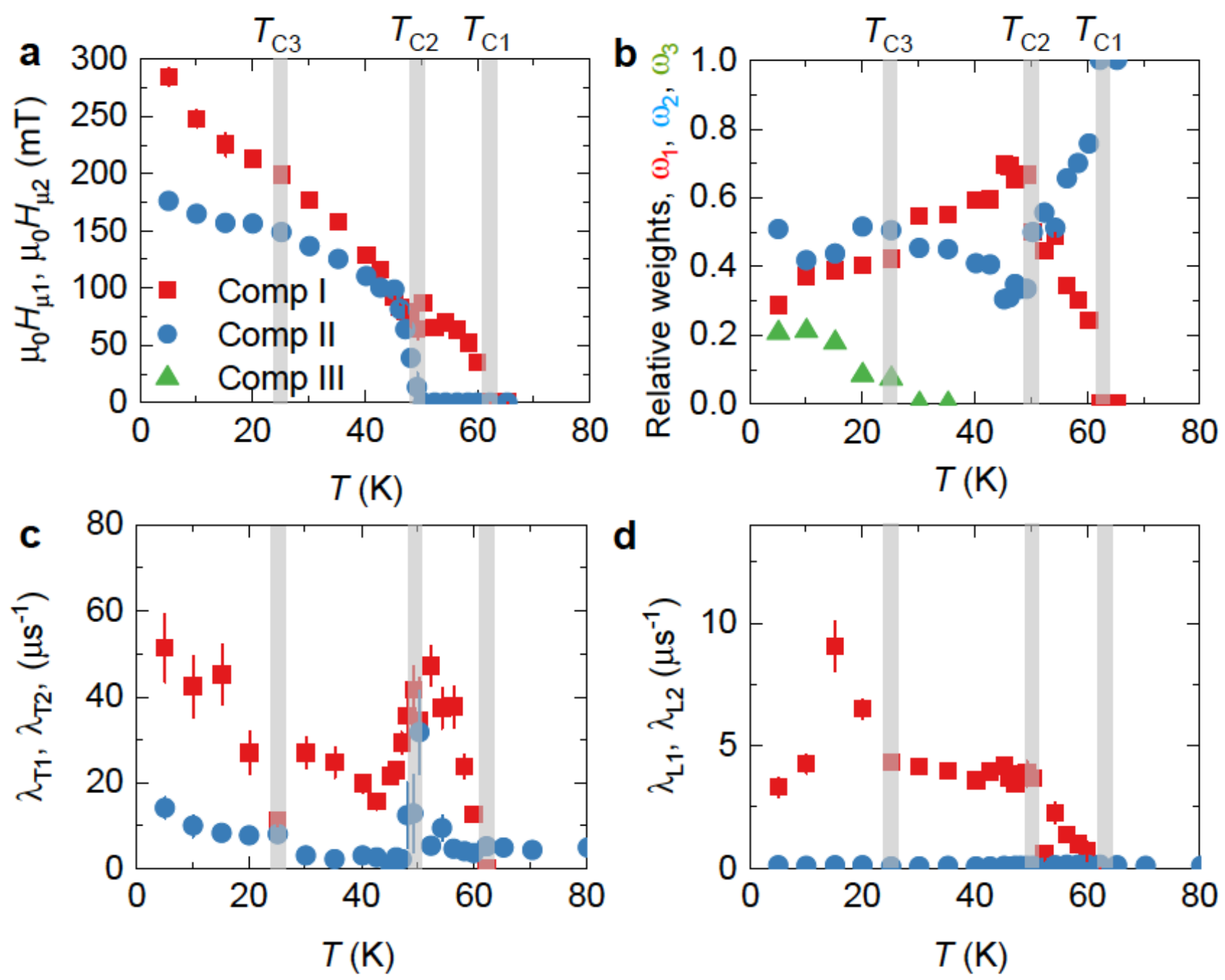

Figure 2

Figure 2

Temperature dependent $\mu$ SR parameters. a, The temperature dependence of the internal magnetic fields for the observed two components in Crl3. b, The temperature dependence of the relative weights of the three components in the total signal for $\mathrm{Crl} 3$, determined from zero-field $\mu \mathrm{SR}$ experiments. c-d, The temperature dependence of transverse depolarization rates $\lambda T 1, \lambda T 2$ and the longitudinal depolarization rates $\lambda L 1, \lambda L 2$ for two components. The error bars represent the standard deviation of the fit parameters. 


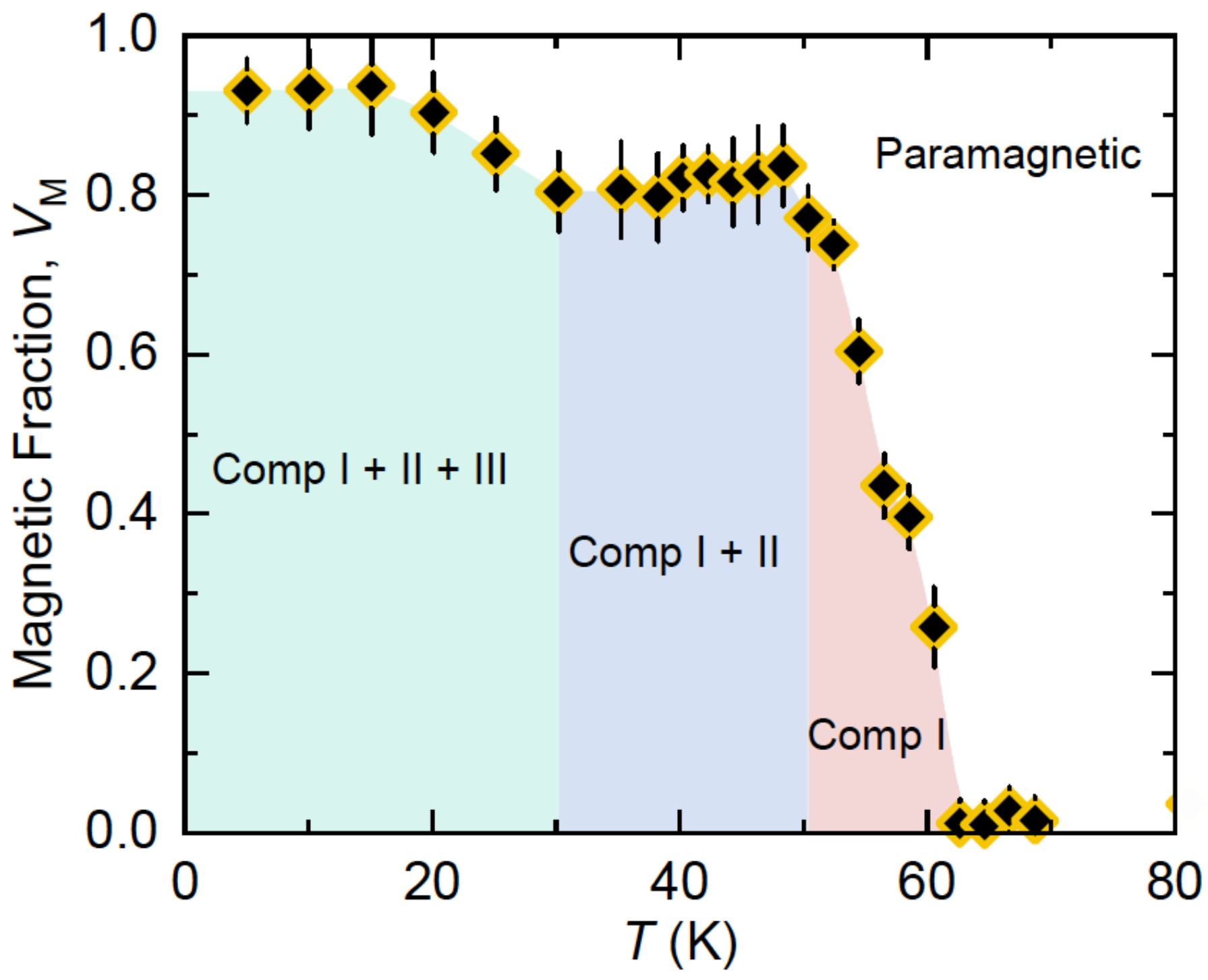

Figure 3

Figure 3

Thermal evolution of various magnetic phases in Crl3. a, The temperature dependence of the total magnetic volume fraction VM, determined from precise weak transverse field (weak-TF) $\mu S R$ measurements. In this weak-TF experiment, a small magnetic field of $30 \mathrm{G}$ is applied nearly perpendicular to the muon spin polarisation. The different components seen in Fig. 2 are highlighted in each region of the temperature range with the paramagnetic phase above the Curie temperature. The error bars represent the standard deviation of the fit parameters. 
a
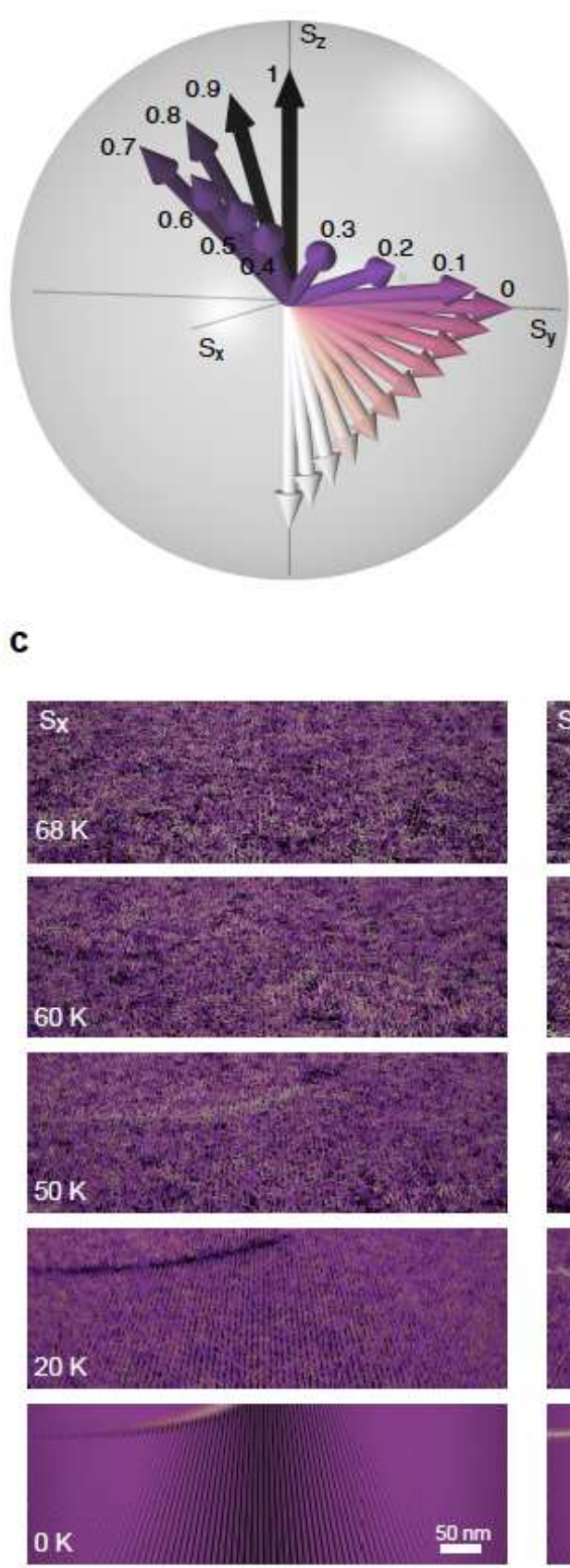

b
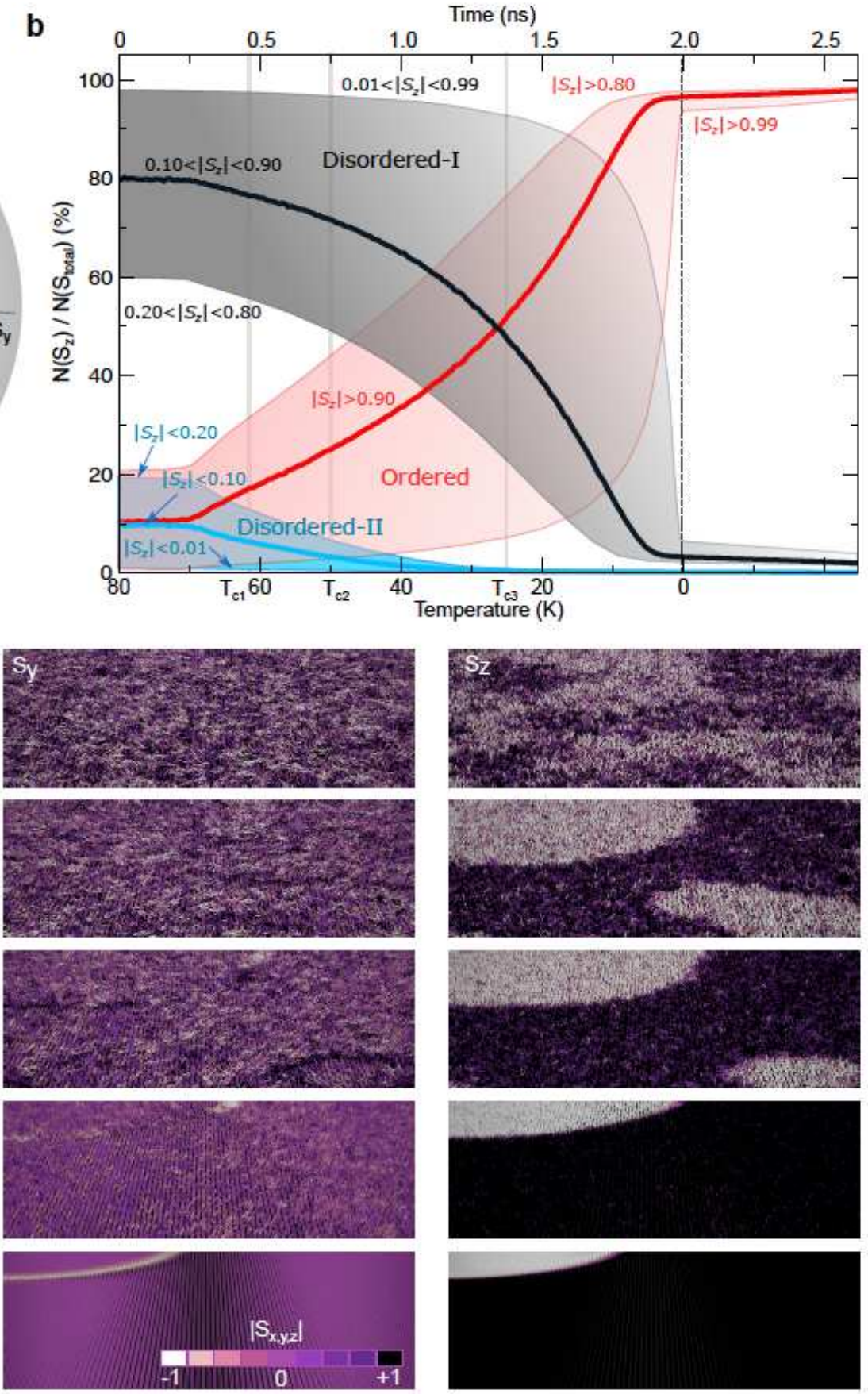

Figure 4

\section{Figure 4}

Micromagnetic analysis of the spin dynamics in Crl3. a, Diagram of the different magnitudes of $|\mathrm{Sz}|$ relative to the unit sphere in the range of $|S z|=1$ (full polarized along $z$ ) and $|S z|=0$ (only in-plane $x y$ projections). The orientations of the spins are assigned randomly following the Monte Carlo moves. The plot only shows a schematic of possible projections over the sphere with others (not shown) with the same $|\mathrm{Sz}|$ equally probable. b, Magnetic fraction (in \%) of the out-of-plane spins $(\mathrm{N}(\mathrm{Sz}) / \mathrm{N}(\mathrm{Stotal}))$ as a function of the temperature. The calculation of $\mathrm{N}(\mathrm{Sz})$ and $\mathrm{N}($ Stotal) take into account the volumetric 
amount of spins over the entire system for Sz and Stotal, respectively. Three main phases were identified in bulk Crl3 which are named: Disordered-I (faint black), with values of $\mathrm{N}(\mathrm{Sz}) / \mathrm{N}(\mathrm{Stotal})$ between the curves of 0:01 $<|\mathrm{Sz}|<0.99$ and $0.20<|\mathrm{Sz}|<0.80$; Ordered (faint red), within $|\mathrm{Sz}|>0.80$ and $|\mathrm{Sz}|>0.99$; and, Disordered-II (faint blue), within $|S z|<0.01$ and $|S z|<0.20$. The solid curves showed in each phase provide a sample of a specific variation of $\mathrm{N}(\mathrm{Sz}) / \mathrm{N}(\mathrm{Stotal})$ for a given range of $|\mathrm{Sz}|$. The vertical dashed line sets the time of $2 \mathrm{~ns}$ required to achieve $0 \mathrm{~K}$ in the spin dynamics. The magnitudes of $\mathrm{N}(\mathrm{Sz}) / \mathrm{N}($ Stotal) showed after $0 \mathrm{~K}$ demonstrated that even when the thermal fluctuations are zero, the system is still evolving to stabilize its ground state. The critical temperatures $(\mathrm{TC1}, \mathrm{C} 2, \mathrm{C} 3)$ are also highlighted. c, Snapshots of the dynamical spin configurations of bulk Crl3 during field cooling from $80 \mathrm{~K}$ down to $0 \mathrm{~K}$. Magnetisation is projected along of in-plane components (Sx, Sy) and Sz at different temperatures: $68 \mathrm{~K}, 60 \mathrm{~K}, 50 \mathrm{~K}, 20 \mathrm{~K}$ and $0 \mathrm{~K}$. Each column and row corresponds to a specific projection of the magnetization at a given temperature provided at the far left. The colour scale shows the variation of the three magnetization components (Sx, Sy, Sz) throughout the system. 15 